\section{Drug-Induced Liver Injury (DILI): A Major Challenge}

Drug-induced liver injury (DILI) is the most common cause for acute liver failure in the USA and Europe. DILI is also a leading cause of attrition of compounds in drug development and one of the two most frequent causes for drug withdrawals, restrictions, and project terminations. Whereas liver signals that escape detection during drug approval result in postmarketing restrictions (e.g., pazopanib, temozolomide and flupirtine in 2013), the risk of falsepositive DILI adjudication may lead to unnecessary attrition, thereby contributing to the considerable economic issues associated with DILI [1].

It is crucial to discriminate two types of DILI, intrinsic and idiosyncratic DILI.

Intrinsic DILI is dose-dependent and occurs with a rather short latency. Prime example for intrinsic DILI is acetaminophen (APAP). Patients who consume APAP at a single dose exceeding $7.5 \mathrm{~g}$ experience acute liver toxicity. Intrinsic DILI is reproducible in preclinical models and much insight has been gained into the underlying mechanisms.

In contrast to intrinsic DILI, the occurence of idiosyncratic DILI, which is very rare but nonetheless responsible for about 10-15\% of acute liver failures in the USA, is almost impossible to predict. This type of DILI is characterised by a variable latency to onset (weeks to months) and a lack of clear dose-dependency. Idiosyncratic DILI may be triggered by many drugs and herbal and dietary supplements and is a diagnostic challenge due to the lack of an in vitro test $[1,2]$. Diagnosis is based on exclusion of other causes of acute liver injury, scoring systems such as RUCAM, drug signatures which can be found in registries such as livertox.nih.gov [3], and on expert adjudication.

No specific treatment has been established for idiosyncratic DILI. Severe cases of acute liver failure may need liver transplantation. The immediate consequence upon diagnosis of DILI is withdrawal of the causal drug. Unfortunately, identification of the culprit drug may be impossible in polypharmacy which is typical in DILI patients. Moreover, the differential diagnosis of autoimmune hepatitis is a big practical challenge with major therapeutic implications [4].

Whereas longterm steroid treatment is key for autoimmune hepatitis it has no proven benefit in DILI but still is fraught with the well known side effects.

On this background, diagnostic and prognostic biomarkers of DILI are urgently needed. Several serum markers and an in vitro causality test based on blood cells of individual affected patients are currently under investigation [5].

\section{Conflict of Interest}

MetaHeps Gmbh, Martinsried, Germany, cofounder and shareholder. EU H2020 Innovative Medicines Initiative, Translational Safety Biomarker Pipeline (TransBioLine) Consortium, LMU Coordinator and Representative for Work Package 2. Pro-Euro-DILI Registry, PI for University Hospital, LMU Munich.
Author

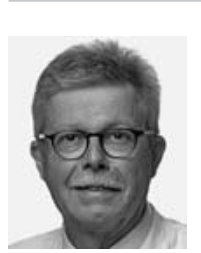

Alexander L. Gerbes ${ }^{(1)}$

MD, Department of Medicine II, Liver Centre Munich, University Hospital, LMU Munich, Germany

Correspondence

Alexander L. Gerbes, MD
Department of Medicine II
Liver Centre Munich
University Hospital
LMU Munich
Marchioninistr. 15
81377 Munich
Germany
gerbes@med.uni-muenchen.de

\section{References}

[1] Kullak-Ublick GA, Andrade RJ, Merz M et al. Drug-induced liver injury: recent advances in diagnosis and risk assessment. Gut 2017; 66: 1154-1164

[2] Chalasani NP, Maddur H, Russo MW et al. ACG Clinical Guideline: Diagnosis and Management of Idiosyncratic Drug-Induced Liver Injury. Am J Gastroenterol 2021; 116: 878898

[3] Bethesda (MD): National Institute of Diabetes and Digestive and Kidney Diseases [Internet]. LiverTox: Clinical and Research Information on Drug-Induced Liver Injury. Available from (accessed 25.08.2021): https://www. ncbi.nlm.nih.gov/books/NBK547852/

[4] Weber S, Benesic A, Rotter I et al. Early ALT response to corticosteroid treatment distinguishes idiosyncratic drug-induced liver injury from autoimmune hepatitis. Liver Int 2019; 39: 1906-1917

[5] Benesic A, Rotter I, Dragoi D et al. Development and validation of a test to identify drugs that cause idiosyncratic drug-induced liver injury. Clin Gastroenterol Hepatol 2018; 16: 1488-1494 